\title{
Ahmadou Kourouma, entre poétique romanesque et littérature politique, dir. P. Voisin
}

\section{Maha Abid-Ben Meftah}

\section{(2) OpenEdition}

10 Journals

\section{Édition électronique}

URL : http://journals.openedition.org/studifrancesi/10616

DOI : 10.4000/studifrancesi. 10616

ISSN : 2427-5856

Éditeur

Rosenberg \& Sellier

\section{Édition imprimée}

Date de publication : 1 décembre 2017

Pagination : 592-593

ISSN : 0039-2944

\section{Référence électronique}

Maha Abid-Ben Meftah, « Ahmadou Kourouma, entre poétique romanesque et littérature politique, dir. P. Voisin », Studi Francesi [En ligne], 183 (LXI | III) | 2017, mis en ligne le 01 février 2018, consulté le 27 janvier 2021. URL : http://journals.openedition.org/studifrancesi/10616 ; DOI : https://doi.org/ 10.4000/studifrancesi.10616

Ce document a été généré automatiquement le 27 janvier 2021.

\section{(c)}

Studi Francesi è distribuita con Licenza Creative Commons Attribuzione - Non commerciale - Non opere derivate 4.0 Internazionale. 


\title{
Ahmadou Kourouma, entre poétique romanesque et littérature politique, dir. P. Voisin
}

\author{
Maha Abid-Ben Meftah
}

\section{RÉFÉRENCE}

Ahmadou Kourouma, entre poétique romanesque et littérature politique, sous la direction de Patrick voIsIN, Paris, Éditions Classiques Garnier, 2015, «Rencontres», 419 pp.

1 Ahmadou Kourouma, entre poétique romanesque et littérature politique est un volume collectif édité sous la direction du professeur Patrick Voisin. L'intention première de ces études réunissant dix-huit contributeurs qui ont fait le choix de se focaliser sur l'œuvre inaugurale de Kourouma, Les Soleils des Indépendances, est de reposer cette question essentielle relative à l'œuvre de l'auteur ivoirien: celle-ci est-elle porteuse d'un engagement, politique ou social ou est-ce plutôt une œuvre tournée vers la recherche formelle d'un impératif esthétique, voire poétique, au service de la création romanesque? En guise d'avant-propos, Patrick Voisin définit clairement la ligne directrice du volume. Il reconnait d'abord l'existence d'un préjugé éditorial et universitaire tenace, celui qui refuse à Kourouma d'être classé parmi les auteurs classiques français. La tradition le relègue ainsi dans le carcan francophone sinon postcolonial, histoire de marquer, par rapport à l'Hexagone, une singularité qui demeure à identifier. Partant de ce constat, Voisin s'interroge ensuite sur la validité de la thèse accolant à Kourouma l'étiquette d'auteur engagé au sens sartrien du terme. Il est vrai qu'un certain discours critique a longtemps insisté sur la fonction testimoniale et contestatrice de son œuvre, en mettant en avant un contexte historique qui permet de justifier l'abandon de la rigueur du discours esthétique au profit d'une lecture référentielle reliant le fonctionnement du texte à sa relation avec le monde réel et occultant de la sorte le fait littéraire. S'appuyant sur le concept de contre-engagement avancé par Benoit Denis, il est possible en revanche selon Voisin de proposer une autre 
posture de lecture récusant toute notion d'engagement étroit car, affirme-t-il, ce texte est un «Roman-hypothèses plutôt que roman-thèse» (p. 17). En d'autres termes, nous sommes face à un romancier qui considère que l'écriture est d'abord un instrument de figuration de l'Histoire cherchant non à la reproduire mais à déclencher chez le lecteur une prise de conscience des situations d'anarchie représentées.

2 L'histoire éditoriale du roman de Kourouma témoigne elle-même de cette inflexion. Rédigé par un mathématicien de formation venu par hasard à la littérature, le tapuscrit est lu par le professeur Vachon à l'occasion d'un concours à Montréal. Ce dernier a su convaincre Kourouma d'abandonner un projet de départ animé par une forte intention savante et chargé de détails politiques alourdissant la fiction pour développer une intrigue autour d'une figure centrale et produire ainsi une écriture doublement subversive au niveau du fond et de la forme. Cela lui a permis d'attirer l'attention et de renouveler le regard de la critique sur un texte qui va devenir un marqueur de rupture dans le rapport à la langue française entretenu par les écrivains francophones. En effet, à une littérature africaine se caractérisant largement par des formes et des contenus occidentaux et se moulant dans une prosodie classique succèdent des textes, à l'image de ceux de Kourouma, portant un intérêt majeur aux questions langagières et recherchant des formes permettant de s'affranchir de la tutelle du modèle occidental. Ce changement dans le rapport à la langue du colonisé, c'est-à-dire le français, apparait en soi comme une sorte de rupture idéologique et conceptuelle dans le paysage littéraire africain, après la publication de ce roman. À la lumière de ce qui précède, on peut conclure à l'existence de deux tendances majeures qui ont largement influencé le travail des chercheurs intervenant dans la rédaction de cet ouvrage. La première tendance est plutôt centrée autour d'une démarche thématique insistant sur le caractère engagé de ce roman inaugural. À cet égard, Bessem ALOUI, Amina AZZA BEKKAT ou encore Habiba FELLAH JEMMALI considèrent dans leurs analyses respectives que l'approche scripturale de Kourouma est à appréhender dans sa relation à un référent historique. C'est un texte qui donne la voix à ceux qui n'ont pas de voix, à un peuple effacé, écrasé par la misère et la violence. L'écriture en prise avec le politique est contrainte d'être engagée face à cette réalité abjecte. D'autres contributeurs vont à leur tour se poser la question de l'inscription du politique dans le roman. Bi Kacou Parfait DIANDUÉ interroge dans une étude géocritique la notion de colonisation, marqueur de l'histoire africaine et thématique majeure des littératures du Sud. Fama devient ainsi l'archétype de la déchéance d'une société menacée par le chaos. Sana DAHMANI le rejoint dans ce sens, elle qui reprend à son tour le parcours tragique de Fama, personnage connaissant le déclassement et devenant le symbole d'une ère de désenchantement, d'une Afrique aux prises avec un nouvel ordre postcolonial voué à l'échec. En revanche, Tanella BONI et Arlette CHEMAIN ont porté leur analyse sur la figure féminine marquante de ce roman, à savoir Salimata. Représentée comme un corps violenté au quotidien, elle est là pour dénoncer le sort de ses compatriotes vivant dans des conditions insoutenables. C'est d'ailleurs ce leitmotiv de la violence couplé au tragique que vont exploiter Ines MOATAMRI et Romain VIGNEST, en analysant le référent spatial à travers le paysage ou les détails météorologiques, témoins du monde dégradé et dégradant dans lequel évoluent les personnages. Dans leur sillage, Affoué Virginie KONANDRI et Roger CHEMAIN appliquent les grilles de la mytho-critique, Abel кOUvouAmA, celles de l'anthropologie, afin de sonder l'ambiance de tension et de destruction où baigne le roman, ambiance qui se propage jusque dans le domaine du rêve et par l'utilisation 
d'un bestiaire qui permet de mieux comprendre le rôle de l'imaginaire dans cette démarche d'historisation. La deuxième tendance qui se dessine chez les contributeurs à cette étude est celle qui considère que le passage à l'écrit pour Kourouma est un acte de reconfiguration du monde. L'auteur se réinvente alors dans l'espace du dire face à un réel qui peut opposer ses limites et son arbitraire. Jean-Pierre FEWOU NGOULOURE a ainsi rappelé que cette langue utilisée par Ahmadou Kourouma en a choqué plus d'un, par sa façon délibérée de transgresser les normes du bon usage mais que la critique a su dépasser l'idée du style dit fautif, et a reconsidéré la question de la norme et de l'écart dictée par un centre prescriptif. En somme, par son refus de la rectitude littéraire, ce roman a participé à la déconstruction d'un système de valeurs érigé en code inviolable. Lobna MESTAOUI estime par conséquent que nous avons affaire à un roman placé sous le signe de l'hybridité. Il s'agit en fait d'un roman dialogique qui préfère à la linéarité narrative l'éclatement du récit et la pluralité des voix au niveau énonciatif. Djédjé Hilaire BонU aborde quant à lui la notion d'hybridité dans son versant linguistique en pointant l'hétérogénéité de la langue du texte avec cette contamination constante du français par les ivoirismes. À l'intérieur de schémas préexistants dans la langue française viennent alors se couler métaphores, proverbes et expressions locales qui l'entraînent vers une expression nouvelle fonctionnant comme une forme de revendication identitaire. Ferroudja ALLOUACHE, Fadhila LAOUANI et Marion MAS insistent enfin sur le fait que face à un réel meurtri, Kourouma a choisi de recourir à l'humour, à l'ironie et au burlesque. Ces divers procédés livrent une configuration du politique propre à déconstruire puis à reconstruire et aident à opérer la distanciation critique nécessaire pour s'approprier l'Histoire.

3 En définitive, les grandes œuvres échappent à leur époque, l'essentiel résidant dans cette créativité qui amène à produire une écriture subversive caractérisée par le déplacement des codes génériques et des normes linguistiques. Kourouma a acquis de la sorte cette stature universelle lui permettant d'avoir sa place du côté des Rabelais, Hugo, Céline et tant d'autres, affirme Patrick Voisin pour conclure. 